Pierre-Yves Marcy

Jean Palussière

Bertrand Descamps

Nicolas Magné

Catherine Ciais

Jean-Noël Bruneton
Pierre-Yves Bondiau

\section{Percutaneous cementoplasty for pelvic bone metastasis}

\section{Support Care Cancer (2000) 8:500-503}

The online version of the original article can be found at http://dx.doi.org/10.1007/ s005200000138

P.-Y. Marcy $(\varangle) \cdot$ B. Descamps N. Magné · P.-Y. Bondiau - C. Ciais J.-N. Bruneton

Centre Antoine-Lacassagne, 33 Avenue

Valombrose, 06189 Nice Cedex 2, France E-mail:

pierre-yves.marcy@cal.nice.fnclcc.fr

Phone: +33-4-92031181

J. Palussière

Institut Bergognié, 33000 Bordeaux,

France
The third author was omitted.

His name and address are as given here: Bertrand Descamps, Centre Antoine-Lacassagne, 33 Avenue Valombrose, 06189 Nice Cedex 2, France. 